\title{
A Simple Technique for Descemet Membrane Exposure in Deep Anterior Lamellar Keratoplasty Using the Transconjunctival Single- plane Sclerocorneal Incision
}

Takahiko Hayashi ( $\nabla$ takamed@gmail.com)

Nihon University School of Medicine

Satoru Yamagami

Nihon University School of Medicine

\section{Research Article}

Keywords: Deep Anterior Lamellar Keratoplasty, big-bubble, learning curve, layer-by-layer, double anterior chamber, transconjunctival single-plane sclerocorneal incision, sclerocorneal incision

Posted Date: April 26th, 2021

DOI: https://doi.org/10.21203/rs.3.rs-446891/v1

License: @ (1) This work is licensed under a Creative Commons Attribution 4.0 International License. Read Full License 


\section{Abstract}

Background: To describe a simple technique for Descemet membrane exposure during deep anterior lamellar keratoplasty (DALK).

Methods: As a prospective interventional case series, consecutive patients who underwent DALK were enrolled. The technique involved the separation of the Descemet membrane by injecting air and ophthalmic viscosurgical devices from the point where transconjunctival single-plane sclerocorneal incision with the slit knife is terminated prior to perforation using a mirror image. Descemet membrane exposure success rates and the incidence of intra- and postoperative complications were analyzed. Outcomes of successful DALK procedures, which were not converted to penetrating keratoplasty, were analyzed by evaluating best corrected visual acuity (BCVA; converted to logarithm of the minimum angle of resolution [logMAR]) and graft survival.

Results: We included 11 eyes from 11 patients (seven males and four females; $53.6 \pm 16.1$ years; mean follow-up duration, 26.1 weeks [6-51 weeks]). Ten surgeries were successful in Descemet membrane exposure without rupture, eight were successful in big-bubble formation, and two were finally successful in Descemet membrane exposure after a failed big-bubble. The DALK procedure for only one eye was converted to a penetrating keratoplasty owing to a tear in the Descemet membrane. BCVA improved from $0.83 \pm 0.38$ logMAR preoperatively to $0.24 \pm 0.12 \log$ MAR postoperatively $(n=10, p<0.001)$. There was no primary graft failure.

Conclusion: This simple technique of transconjunctival single-plane sclerocorneal incision could be very useful for exposing the Descemet membrane during DALK.

\section{Background}

Since the development of the first penetrating keratoplasty (PKP), corneal transplantation has been the most common type of organ transplantation. ${ }^{1,2}$ Despite the dramatic improvement in the complete replacement of severe corneal opacity, there are some downsides to PKP such as suture-related problems (higher astigmatism or infection), transplant rejection, glaucoma (steroid-dependent), and rupture due to injury. ${ }^{3}$

Over the last decade, PKP has evolved rapidly with the increasing number of lamellar surgeries such as deep anterior lamellar keratoplasty (DALK) and endothelial keratoplasty (EK) ${ }^{3,4}$ Particularly, simple stromal opacity without endothelial dysfunction can be treated with DALK. ${ }^{5,6}$ DALK has certain advantages over PKP or EK as it causes less endothelial cell density (ECD) reduction and low immunological graft rejection rates. ${ }^{7}$ The greatest difficulty in performing DALK is the surgical learning curve. Anwar's big-bubble technique and the Melles technique are two major methods to achieve Descemet membrane exposure in order for surgeons to perform DALK quickly and easily; however, the incision depth is difficult to determine, and these techniques are not reproducible. ${ }^{8-11}$ Even an experienced surgeon risks failure of the procedure due to an incomplete Descemet membrane exposure or rupture. ${ }^{12-13}$

This case series investigated a simplified technique using transconjunctival single-plane sclerocorneal incision, which is familiar to cataract surgeons as well as corneal surgeons. ${ }^{14}$ The purpose of this study was to render a less steep surgical learning curve for DALK, especially Descemet membrane exposure.

\section{Methods}

\section{Study Design}

This prospective study was performed in accordance with the institutional guidelines, and all the patients had provided their informed consent. This study adhered to the tenets of the Declaration of Helsinki and was approved by the Institutional Review Board (Yokohama Minami Kyosai Hospital Ethical Review Board, Approval no. YKH_26_05_12). Consecutive patients requiring DALK from July 2018 to Mar 2019 were enrolled. Patients with a history of corneal perforation prior to surgery, ocular surgery, or any pre-existing diseases such as macular degeneration, amblyopia, and advanced glaucoma were excluded from this study.

\section{Surgical Technique}

All surgeries were performed under retrobulbar anesthesia and the Nadbath facial nerve block. All surgical steps are shown in Fig. 1.

Firstly, the host cornea was marked using a trephine (Katena, Denville, USA) at $7.5 \mathrm{~mm}$. Without a superficial keratectomy, a sclerocorneal pocket was created using a 2.8-mm slit knife, similar to that in a cataract surgery, without perforation as shown in 
Fig. 1A. The most important feature is the incision depth; the incision has to be stopped just prior to the perforation (Fig. 1B). Although this process seems intuitive, the incision depth can be judged by the shape of the mirror image or wrinkle of the Descemet membrane. The intraoperative spectral domain Optical coherence tomography (OCT) (RESCAN 700 [Carl Zeiss Meditec, Germany]) was used to check the depth of the incision. A small slit was created with a disposable knife, and air was injected using a 30-gauge needle (bigbubble formation) at a bevel-down orientation ${ }^{9}$ to completely separate the host stroma and the Descemet membrane (Fig. 1C, 1D), while ophthalmic viscosurgical devices (OVD) (OPELEAD ${ }^{\circledR}$ HV [0.85] 1\%) were used to create a space by depressing the Descemet membrane. The anterior stroma was divided into four squares. (Fig. 1E). The host stroma was carefully removed as was the donor endothelium, and the DALK graft was prepared using a donor punch (Katena, Denville, USA) at $7.75 \mathrm{~mm}$. The donor graft was sutured using $10-0$ nylon (Mani, Tochigi, Japan) by either a running or an interrupted suture technique (Fig. 1F).

\section{Postoperative Care}

Postoperative medications administered were $1.5 \%$ levofloxacin (Cravit ${ }^{\circledR}$ ), $0.1 \%$ betamethasone sodium phosphate (Sanbetasone ${ }^{\circledR}$; Santen Pharmaceutical Co., Ltd., Osaka, Japan), and 2\% rebamipide ophthalmic solution (Mucosta ${ }^{\circledR}$; Otsuka Pharmaceutical CO., Ltd., Japan, Tokyo), starting at four times per day for three months and subsequently tapered. In cases of postoperative glaucoma, antiglaucoma agents were applied.

\section{Patients and Examinations}

The evaluated outcomes were as follows: Best corrected visual acuity (BCVA) (converted to logarithm of the minimal angle of resolution [logMAR] units) pre- and postoperatively (at the last visit after surgery), astigmatism (diopters [D]) postoperatively (last visit after surgery), success rates of Descemet membrane exposure, and incidence of intra- and postoperative complications. Astigmatism was assessed using the autorefractometer ARK-1 (Nidek Co., Ltd, Japan). The main outcome results for all included cases are shown in Table 1. Cases of conversion to PKP were excluded from postoperative clinical course evaluations such as BCVA, corneal astigmatism, and postoperative complications.

Table 1

Patient Characteristics

\begin{tabular}{|c|c|c|c|c|c|c|c|c|c|c|}
\hline Case & Sex & OD/OS & $\begin{array}{l}\text { Surgical } \\
\text { Indication }\end{array}$ & $\begin{array}{l}\text { Type } \\
\text { of } \\
\text { surgery }\end{array}$ & $\begin{array}{l}\text { Successful } \\
\text { Big-bubble } \\
\text { (Yes/ No) }\end{array}$ & Rebubbling & $\begin{array}{l}\text { Pre } \\
\text { BCVA }\end{array}$ & $\begin{array}{l}\text { Post } \\
\text { BCVA }\end{array}$ & $\begin{array}{l}\text { Astigmatism } \\
\text { (D) }\end{array}$ & $\begin{array}{l}\text { Observation } \\
\text { period } \\
\text { (Weeks) }\end{array}$ \\
\hline 1 & $\mathrm{~F}$ & OS & Scar & DALK & Yes & Yes & $20 / 150$ & $20 / 50$ & -2 & 51 \\
\hline 2 & $M$ & os & Dystrophy & DALK & Yes & No & $20 / 100$ & $20 / 25$ & -4.5 & 50 \\
\hline 3 & M & OD & Dystrophy & DALK & Yes & Yes & $20 / 50$ & $20 / 25$ & -4.5 & 20 \\
\hline 4 & M & OD & Scar & DALK & No & No & $20 / 60$ & $20 / 40$ & -8 & 16 \\
\hline 5 & M & OD & Keratoconus & DALK & Yes & No & $20 / 400$ & $20 / 50$ & -2 & 16 \\
\hline 6 & M & OD & Dystrophy & DALK & Yes & No & $20 / 60$ & $20 / 25$ & -1.5 & 35 \\
\hline 7 & $\mathrm{~F}$ & OD & Scar & DALK & No & No & $20 / 50$ & $20 / 25$ & -3 & 6 \\
\hline 8 & $\mathrm{~F}$ & os & Scar & DALK & Yes & No & $20 / 150$ & $20 / 30$ & -3.75 & 8 \\
\hline 9 & M & OS & Keratoconus & DALK & Yes & No & $20 / 500$ & $20 / 30$ & -3 & 10 \\
\hline 10 & $\mathrm{~F}$ & OS & Scar & DALK & Yes & Yes & $20 / 500$ & $20 / 25$ & -1.5 & 49 \\
\hline 11 & $M$ & OD & Scar & PKP & $\begin{array}{l}\text { No } \\
\text { (Rupture of } \\
\text { the } \\
\text { Descemet } \\
\text { membrane) }\end{array}$ & No & $20 / 500$ & $20 / 50$ & -3.75 & 6 \\
\hline
\end{tabular}




\section{Statistical analysis}

We performed the statistical analyses using JMP Pro software version 14.0.0 (SAS Institute, Cary, NC, USA). Statistical significance was defined as $p<0.05$. All data were described as mean \pm standard deviation. For the statistical analyses, BCVA was converted to logMAR units. The Mann-Whitney U test was used to compare the preoperative and postoperative outcomes, namely BCVA and astigmatism.

\section{Results}

\section{Patient Characteristics}

Four female and seven male patients were included in this study, with an average age of $53.6 \pm 16.1$ years. Surgical indications were scars $(n=6)$, corneal dystrophy $(n=3)$, or keratoconus $(n=2)$. One was excluded from the postoperative analysis of the clinical course owing to the conversion to a PKP. The average follow-up period was $26.1 \pm 16.3$ weeks (Table 1 ).

\section{Clinical course}

BCVA significantly improved from $0.83 \pm 0.38 \log M A R$ at 20/150 Snellen baseline preoperatively to $0.24 \pm 0.12 \log$ MAR at 20/35 Snellen $(n=10, p<0.001)$ postoperatively. Postoperative astigmatism was $-3.3 \pm 1.9 D(n=10)($ Table 1$)$.

\section{Complications}

Except for one case of unsuccessful separation (one eye), Descemet membrane exposure was successful in ten of the 11 cases (90.9 $\%$ ) without perforation. One eye was unsuccessful due to a large tear in the Descemet membrane that made it impossible to complete the DALK and necessitated conversion to a PKP. Three out of ten (30\%) patients who underwent a DALK showed double chamber formation, wherein spontaneous attachment was obtained. There was no graft rejection or graft failure observed during the follow-up period (Table 1).

\section{Discussion}

Our study demonstrated the use of a simple technique, transconjunctival single-plane sclerocorneal incision, which is familiar to ophthalmic surgeons and cataract surgeons, to achieve Descemet membrane exposure. Our technique allowed high success rates (up to $72.7 \%$ [eight of 11 eyes]) in performing the big-bubble technique. In cases of failure, we were still able to completely expose the Descemet membrane using an additional OVD injection. Unfortunately, one eye showed Descemet membrane rupture that required conversion to a PKP.

Previous studies have introduced some novel techniques, such as the layer-by-layer technique (manual dissection), ${ }^{11}$ big-bubble technique, ${ }^{9}$ divide-and-conquer technique ${ }^{15}$ double-bubble technique, ${ }^{16}$ mirror-image technique, ${ }^{8,17}$ and limbal-approach technique. ${ }^{18}$ In all the cases, every method has a steep learning curve. For instance, increased success of the big-bubble technique is obtained with mastery of the skill. Incidentally, it has been demonstrated that DALK success rates significantly decrease with the surgeons' experience. $^{12,13}$

The strength of our technique is its high reproducibility $(72.7 \%)$, which could be attributed to the depth of the needle injection. In our technique, the depth of insertion could be easily determined by a mirror image or an irregular wrinkle of the Descemet membrane. Although the depth of the slit knife incision can be determined by intuition, only ophthalmic surgeons with experience in performing cataract surgeries may have developed this acuity. In fact, the use of intraoperative OCT revealed that the depth of the first incision with the slit knife or needle injection is adequate to create a big-bubble.

There were certain limitations to this study, such as the relatively small number of participants, and the lack of a comparison with a control group including other techniques of Descemet membrane exposure such as manual DALK OVD techniques. In our case series, we used OVD in all the eyes to depress the Descemet membrane. The relatively high rates of double anterior chamber formation (30\%) could be attributed to the use of OVD. Despite the possibility of spontaneous attachment of the host-Descemet membrane, ${ }^{19}$ it is important to wash OVD well at the end of surgery. 
In conclusion, the current study advocates the efficacy of transconjunctival single-plane sclerocorneal incisions for Descemet membrane exposure in DALK.

\section{List Of Abbreviations}

BCVA, best corrected visual acuity ; D, diopter; DALK, deep anterior lamellar keratoplasty; ECD, endothelial cell density; EK, endothelial keratoplasty; logMAR, logarithm of the minimum angle of resolution; OVD, ophthalmic viscosurgical device; PKP, penetrating keratoplasty

\section{Declarations}

\section{Ethics approval and consent to participate}

This study was approved by the Institutional Review Board (Yokohama Minami Kyosai Hospital Ethical Review Board, Approval no. YKH_26_05_12). All patients provided informed consent for participation.

\section{Consent for publication}

All patients provided informed consent for publication.

\section{Availability of data and materials}

The datasets used and/or analysed during the current study available from the corresponding author on reasonable request.

\section{Competing interests}

No.

\section{Funding}

This work was supported by the Japan Eye Bank Association (http://www.j-eyebank.or.jp).

\section{Authors' contributions}

T.H. wrote the manuscript. S.Y. organized the paper. All authors reviewed the paper.

\section{Acknowledgements}

The authors thank Dr. I Oyakawa for providing critical comments concerning this study.

\section{References}

1. Zirm EK. Eine erfolgreiche totale keratoplastik (A successful total keratoplasty). Arch Fr Ophthalmol. 1906;64:580-583.

2. Coster DJ, Williams KA. The impact of corneal allograft rejection on the long-term outcome of corneal Am J Ophthalmol. 2005;140:1112-1122.

3. Hos D, Matthaei M, Bock F, et al. Immune reactions after modern lamellar (DALK, DSAEK, DMEK) versus conventional penetrating corneal transplantation. Prog Retin Eye Res. 2019;100768.

4. Flockerzi E, Maier P, Böhringer D, et al. Trends in Corneal Transplantation from 2001 to 2016 in Germany: A Report of the DOGSection Cornea and its Keratoplasty Registry. Am J Ophthalmol. 2018;188:91-98.

5. Reinhart WJ, Musch DC, Jacobs DS, et al. Deep anterior lamellar keratoplasty as an alternative to penetrating keratoplasty a report by the American academy of ophthalmology. Ophthalmology. 2011;118:209-218.

6. Ple-Plakon PA, Shtein RM. Trends in corneal transplantation: indications and techniques. Curr Opin Ophthalmol. 2014;25:300-305.

7. Ogawa A, Yamaguchi T, Mitamura H, et al. Aetiology-specific comparison of long-term outcome of deep anterior lamellar keratoplasty for corneal diseases. Br J Ophthalmol. 2016;100:1176-1182. 
8. Melles GRJ, Lander F, Rietveld FJR, et al. A new surgical technique for deep stromal, anterior lamellar keratoplasty. Br J Ophthalmol. 1999;83:327-333.

9. Anwar M, Teichmann KD. Big-bubble technique to bare Descemet's membrane in anterior lamellar keratoplasty. J Cataract Refract Surg. 2002;28:398-403.

10. Shimazaki J, Shimmura S, Ishioka M, et al. Randomized clinical trial of deep lamellar keratoplasty vs penetrating keratoplasty. Am J Ophthalmol. 2002;134:159-165.

11. Sugita J, Kondo J. Deep lamellar keratoplasty with complete removal of pathological stroma for vision improvement. $\mathrm{Br} \mathrm{J}$ Ophthalmol. 1997;81:184-188.

12. Sarnicola V, Toro P, Gentile D, et al. Descemetic DALK and predescemetic DALK: outcomes in 236 cases of keratoconus. Cornea. 2010;29: 53-59.

13. Smadja D, Colin J, Krueger RR, et al. Outcomes of deep anterior lamellar keratoplasty for keratoconus: learning curve and advantages of the big bubble technique. 2012;31:859-863.

14. Sugai S, Yoshitomi F, Oshika T. Transconjunctival single-plane sclerocorneal incisions versus clear corneal incisions in cataract surgery. J Cataract Refract Surg. 2010;36:1503-1507.

15. Tsubota $\mathrm{K}$, Kaido $\mathrm{M}$, Monden $\mathrm{Y}$, et al. A new surgical technique for deep lamellar keratoplasty with single running suture adjustment. Am J Ophthalmol. 1998;126:1-8.

16. Shimazaki J. Double-bubble technique to facilitate Descemet membrane exposure in deep anterior lamellar keratoplasty. $J$ Cataract Refract Surg. 2010;36:193-196.

17. Shimmura S, Shimazaki J, Omoto M, et al. Deep lamellar keratoplasty (DLKP) in keratoconus patients using viscoadaptive viscoelastics. 2005;24:178-181.

18. Senoo T, Chiba K, Terada O, et al. Deep lamellar keratoplasty by deep parenchyma detachment from the corneal limbs. $\mathrm{Br} J$ Ophthalmol. 2005;89:1597-1600.

19. Lin X, Wu Y, Fu Y, et al. Spontaneous reattachment of Descemet membrane detachment after deep anterior lamellar keratoplasty: A case report. Medicine (Baltimore). 2018;97:e0032.

\section{Figures}

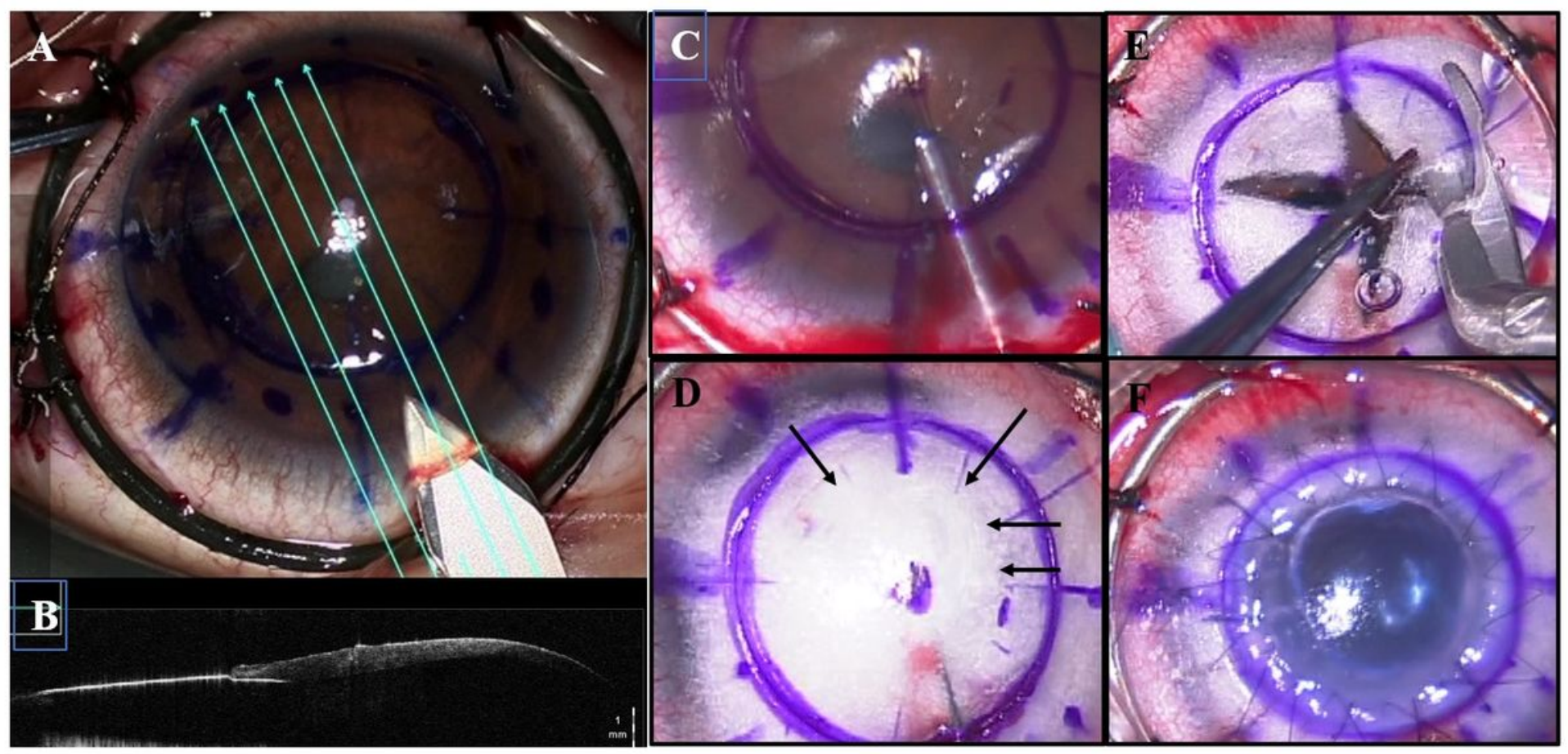

Figure 1 
Limbal approach via a transconjunctival single-plane sclerocorneal tunnel for Descemet membrane exposure in deep anterior lamellar keratoplasty (DALK) (A) A 2.8-mm slit knife is used to create a sclerocorneal pocket, similar to that created during a cataract surgery. Incision depth is shown here by the shape of the mirror image of the Descemet membrane. (B) As shown by an intraoperative optical coherence tomography (OCT), the depth created by the slit knife is $80 \%$ or more of the full corneal thickness, which increases the success rate of big-bubble formation. (C) A dull needle is used to form the tunnel that reaches the central area where the graft should be placed. Air pressure is introduced via the tunnel to separate the Descemet membrane (big-bubble technique). The arrows indicate the separated lines achieved using the big-bubble technique. (D) After the separation of the lines, ophthalmic viscosurgical devices (OVD) are injected to depress the Descemet membrane. (E) Vannas or Katzin scissors are used for the host-stroma removal. (F) After placing four cardinal sutures, the donor graft is sutured. 